\title{
Perturbed period-doubling bifurcation. I. Theory
}

\section{Svensmark, Henrik; Samuelsen, Mogens Rugholm}

\section{Published in:}

Physical Review B

Link to article, DOI:

10.1103/PhysRevB.41.4181

Publication date:

1990

Document Version

Publisher's PDF, also known as Version of record

Link back to DTU Orbit

Citation (APA):

Svensmark, H., \& Samuelsen, M. R. (1990). Perturbed period-doubling bifurcation. I. Theory. Physical Review B, 41(7), 4181-4188. https://doi.org/10.1103/PhysRevB.41.4181

\section{General rights}

Copyright and moral rights for the publications made accessible in the public portal are retained by the authors and/or other copyright owners and it is a condition of accessing publications that users recognise and abide by the legal requirements associated with these rights.

- Users may download and print one copy of any publication from the public portal for the purpose of private study or research.

- You may not further distribute the material or use it for any profit-making activity or commercial gain

- You may freely distribute the URL identifying the publication in the public portal

If you believe that this document breaches copyright please contact us providing details, and we will remove access to the work immediately and investigate your claim 


\title{
Perturbed period-doubling bifurcation. I. Theory
}

\author{
Henrik Svensmark \\ Department of Physics, University of California, Berkeley, Berkeley, California 94720 \\ and Materials and Chemical Sciences Division, Lawrence Berkeley Laboratory, Berkeley, California 94720 \\ M. R. Samuelsen \\ Physics Laboratory I, The Technical University of Denmark, DK-2800 Lyngby, Denmark
}

(Received 24 May 1989)

\begin{abstract}
The influence of perturbations (a small, near-resonant signal and noise) on a driven dissipative dynamical system that is close to undergoing a period-doubling bifurcation is investigated. It is found that the system is very sensitive, and that periodic perturbations change its stability in a welldefined way that is a function of the amplitude and the frequency of the signal. New scaling laws between the amplitude of the signal and the detuning $\delta$ are found; these scaling laws apply to a variety of quantities, e.g., to the shift of the bifurcation point. It is also found that the stability and the amplification of the system are not stationary, but vary with $\delta$. The results in this paper are found from a linear analysis of the dynamics in the Poincare map. It is thereby shown that several effects, which previously were believed to be caused by nonlinearity, are in fact linear in the lowest order. Numerical and analog simulations of a microwave-driven Josephson junction confirm the theory. Results should be of interest in parametric-amplification studies.
\end{abstract}

\section{INTRODUCTION}

Perturbations on a nonlinear system that is close to a period-doubling bifurcation have attracted considerable interest, ${ }^{1-7}$ and have been studied theoretically by looking at either discrete $e^{1,7}$ or continuous dynamical systems. ${ }^{2-6}$ From the point of view of applications, the problem is of particular interest in connection with parametric amplification, for example in Josephson-junction amplifiers, ${ }^{8-11}$ where the fundamental amplifying mechanism is based on the period-doubling bifurcation. Therefore the results of this paper might be of relevance to that problem.

The main concern is the influence of a periodic (coherent) perturbation that is close to parametric resonance frequencies of the unperturbed system; the unperturbed system oscillates with a frequency $\omega_{D}$, and the periodic perturbation is close to the frequencies $\frac{1}{2} \omega_{D}, \omega_{D}, \frac{3}{2} \omega_{D}$. The effect of noise is also addressed here. This paper does not focus on a particular dynamical system but, rather, on the dynamics in phase space of a general system that is close to a period-doubling bifurcation, and the conclusions are therefore expected to be general. The results of this paper are based on the fact that the dynamics of a system at the bifurcation point is confined to a two-dimensional surface in phase space called the center manifold; ${ }^{12}$ this makes a large simplification of the complexity of the dynamics in the vicinity of the bifurcation point. The existence of this center manifold makes it possible to make a simple one-dimensional iterative model describing the dynamics in the Poincare map. ${ }^{2}$ The solution of the model reveals several interesting features of the influence of perturbations on the period-doubling bifurcation; some of these features are described here. Apart from amplification of perturbations close to the bi- furcation point, a periodic perturbation close to the resonance frequencies $\frac{1}{2} k \omega_{D}$, where $k$ is an odd integer, always stabilizes the system against bifurcation. Further, this stabilization, i.e., the shift of the bifurcation point, follows a particular scaling law. This shift is proportional to the square of the amplitude of the periodic perturbation. This result differs from that of Bryant and Wiesenfeldt $^{5}$ who found the shift of the bifurcation point to be proportional to the amplitude of the periodic perturbation to the power $\frac{2}{3}$. It is also found that maximum amplification or maximum gain of a small signal does not coincide with the bifurcation point but occurs slightly below the shifted bifurcation point. A scaling law between the amplitude of the periodic perturbation and the detuning $\delta$ (the relative frequency difference between the periodic perturbation and the parametric resonance frequency) is also found. Surprisingly these results can be found from a linear analysis, thereby showing that the effects are not caused by nonlinearities, as found in previous work. ${ }^{5}$ It should be emphasized that it is in general impossible to determine the actual bifurcation point in the presence of perturbations by an analysis of the power spectrum.

Comparison of the theory with numerical and analog simulations of a microwave-driven Josephson junction shows good agreement. Experiments on a small Josephson junction have also shown agreement with the scaling laws derived in this paper. ${ }^{13}$

Section II contains the theoretical development. In Sec. II A the problem is formulated, and a model for the dynamics in the Poincare map is derived. In Sec. II B the model is solved, and in Secs. II C and II D the results are shown and interpreted. Section III contains the comparison between the theory and the results of numerical and analog simulations of a microwave-driven Josephson junction. Section IV is the conclusion. 


\section{THEORY}

In this section a general equation describing the behavior of a dynamical system close to period-doubling bifurcation is derived. The equation is general, i.e., it is independent of the particular dynamical system. Instead of working in the full phase space of the dynamical system, the dynamics are described in the Poincare section. ${ }^{12}$ Even though this is a large simplification of the complexity of problem, it preserves the interesting main features of the bifurcation. A general dissipative dynamical system is assumed to be modeled by the following differential equation:

$\dot{x}=f_{\mu}(x, t)+s(t)+v(t), \quad(x, t) \in R^{n} \times R, \quad \mu \in I$,

where $f_{\mu}(\cdot, t)=f_{\mu}\left(\cdot, t+T_{D}\right)$ is periodic with the period $T_{D}$. The control parameter $\mu$ is defined in an interval $I$. The functions $s(t)$ and $v(t)$ are a small-signal (periodic perturbation) and a small-noise term (stochastic perturbation), respectively. The period of the signal is denoted $T_{S}$ and the corresponding frequency $\omega_{S}=2 \pi / T_{S}$. The noise term $v(t)$ is assumed to be "white," such that $\langle v(t) v(t+\tau)\rangle=\sigma \delta(\tau)$ and $\langle v(t)\rangle=0$. The perturbation amplitudes $|s(t)|$ and $|\nu(t)|$ are small compared to $\left|f_{\mu}(x, t)\right|$.

The unperturbed system is assumed to be close to a period-doubling bifurcation. This means that by increasing the control parameter $\mu$, the response changes from a periodic solution $\gamma$, with period $T_{D}$, to a periodic solution $\gamma^{*}$, of period $2 T_{D}$. The value $\mu_{0}$ of the control parameter for which this happens is called the bifurcation point. The theory that follows is valid for $\mu$ close to, and smaller than this bifurcation value.

\section{A. Dynamics in the Poincaré map}

The motion governed by the dynamical system is periodic, i.e., the orbits in phase space are closed. Therefore a tool for investigating the behavior of orbits near the closed orbit is the Poincare map. ${ }^{4}$ Here this map is given by a local transverse section of an orbit in phase space at a multiple of the period $T_{D}$. With the aid of the Poincaré map, the dynamical system given by Eq. (1) is transformed into a discrete dynamical system

$$
x_{n+1}=P_{\mu}\left(x_{n}\right), \quad x_{n} \in R^{n}, \quad \mu \in I .
$$

A closed orbit of period $T_{D}$ in phase space corresponds to a fix point in the Poincare map. The stability of the fix point reflects the stability of the closed orbit. Equation (2) can be linearized around the fix point to yield an eigenvector-eigenvalue equation. A period-doubling bifurcation is associated with an eigenvalue $\lambda=-1$ at the fix point $x^{\text {fix }}$ of the Poincaré map. ${ }^{12}$ (All other eigenvalues have, in this case, magnitudes small compared to 1.) This means that an orbit $\gamma^{*}$ alternates from one side of the fix point to the other along the direction of the eigenvector $e_{\lambda=-1}$ (see Fig. 1). In full phase space, orbits similar to $\gamma^{*}$ are all confined to a two-dimensional surface called the center manifold. In the case of the perioddoubling bifurcation the center manifold can be described as a Möbius band ${ }^{12}$ (see Fig. 1). Here it is assumed that

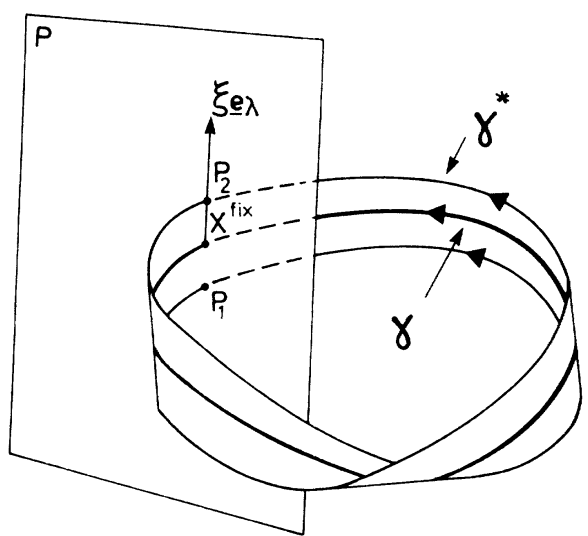

FIG. 1. Intersection of the center manifold of a perioddoubling bifurcation with the Poincare map $P$. The orbit $\gamma$ of period $T_{D}$ is confined to the center manifold and intersects $P$ at the fix point $x^{\mathrm{fix}}$. The vector $e_{\lambda}$ is the eigenvector for the eigenvalue $\lambda=-1$. The orbit $\gamma^{*}$ is periodic with the period $2 T_{D}$, and intersects $P$ at the points $p_{1}$ and $p_{2}$.

all relevant orbits of a dynamical system close to a period-doubling bifurcation are confined to this surface. As a result the Poincaré map becomes one dimensional, regardless of the dimension of the original dynamical system. With this in mind, the stability of the fix point can be analyzed by linearizing the Poincare map around the fix point. Therefore, the stability equation becomes

$$
\xi_{n+1}=\lambda \xi_{n}
$$

where $\xi_{n}$ is the deviation from the fix point $x^{\text {fix }}$ on the center manifold, and $\lambda$ is the corresponding eigenvalue in this direction (see Fig. 1). It is assumed that the control parameter $\mu$ and the eigenvalue $\lambda$ are linearly related close to the bifurcation point. The fix point is stable if $|\lambda|<1$. If $|\lambda|>1$, the fix point becomes unstable and period-doubling bifurcation takes place.

If a small periodic perturbation with the frequency $\omega_{S}$ is introduced $\left[s(t) \neq 0\right.$ in (1)], the function $P\left(x_{n}\right)$ on the right-hand side of (2) changes; it no longer has period $T_{D}$. The Poincaré map can now be written

$$
x_{n+1}=P_{n}^{*}\left(x_{n}\right), \quad x_{n} \in R .
$$

Since the perturbation $s(t)$ is small, (4) can be approximated by

$$
x_{n+1}=P\left(x_{n}\right)+P_{n}^{* *}\left(x_{n}-x^{\mathrm{fix}}\right), \quad x_{n} \in R,
$$

where the first term, $P$, is the unperturbed Poincare map and the second term, $P_{n}^{* *}$, is a small correction due to the period $\omega_{S}$ perturbation. The last term is different for each iteration, as indicated by the subindex $n$. The right-hand side of (1) now contains two frequencies, which yield a periodic or quasiperiodic driving term, depending on the ratio of the frequencies $\omega_{S} / \omega_{D}$. Therefore the perturbation term $P_{n}^{* *}$ in (5), changes from cycle to cycle as determined by the right-hand side of (1). Before writing an explicit expression for $P_{n}^{* *}$, it is useful to look at the two frequencies from a geometrical point of view. 
At a given instant of time, the phase angles corresponding to the driving term $\phi_{D}$ and the signal $\phi_{S}$, the first two terms on the right-hand side of $\mathrm{Eq}$. (1), can be viewed as coordinates on a torus,

$$
\left[\phi_{D}, \phi_{S}\right]=\left[\omega_{D} t(\bmod 2 \pi), \omega_{S} t(\bmod 2 \pi)\right],
$$

as seen in Fig. 2, which illustrates the important case where the ratio $\omega_{S} / \omega_{D}$ is close to one half. After one iteration $\left(\phi_{D}\right.$ change of $\left.2 \pi\right)$ the signal phase angle $\phi_{S}$ increases by $\pi+\pi \delta$; after two iterations it becomes $2 \pi \delta(\bmod 2 \pi)$. This example is easily generalized to include the ratio $\omega_{S} / \omega_{D} \approx 1$, with $\delta$ given by

$$
\delta=2\left(\omega_{S} / \omega_{D}\right)-k
$$

where $k$ is either 0 or 1 , such that $-\frac{1}{2}<\delta<\frac{1}{2}$. In this way $\delta$ is always measured with respect to the nearest resonance frequency, $\omega_{S} / \omega_{D}=\frac{1}{2}$ or $\omega_{S} / \omega_{D}=1$. It should be emphasized that the description given here is also valid for an arbitrary integer value of $k$, corresponding to frequencies $\omega_{S}$ close to the parametric resonance frequencies $\frac{1}{2} \omega_{D} k$.

From the preceding considerations, it follows that the phase of the signal function $s(t)$ on the right-hand side of

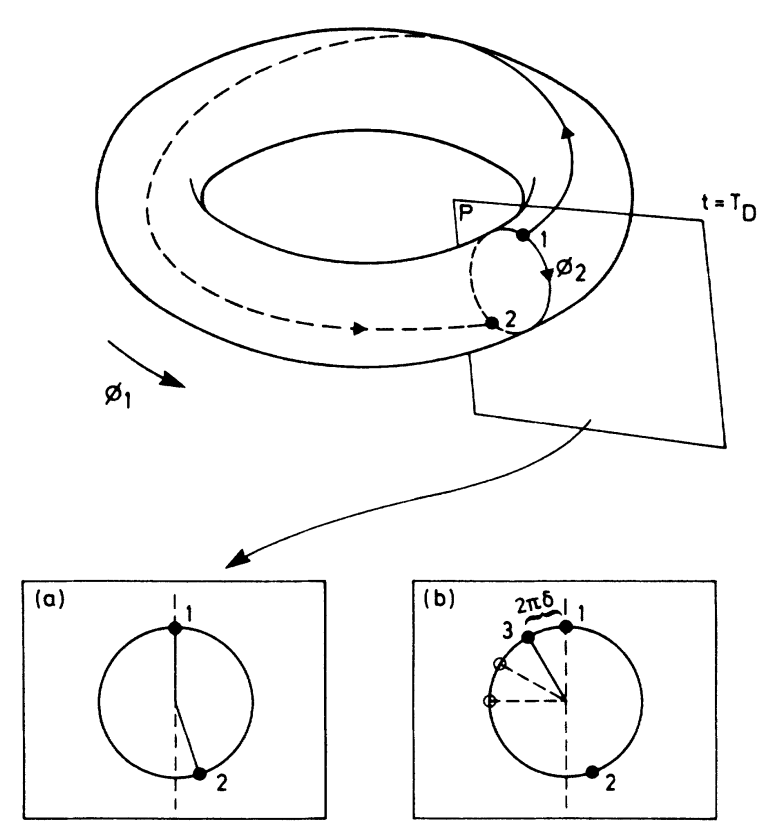

FIG. 2. Geometrical view of the two-phase angles for the two first terms on the right-hand side of Eq. (1). The phase angles are given as coordinates on a torus. The figure shows an example where the periodic perturbation is close to the resonance frequency $\omega_{S} / \omega_{D}=\frac{1}{2}$. (a) shows how the phase $\phi_{s}$ changes after a period $T_{D}$. (b) shows how the phase $\phi_{s}$ changes after an interval $2 T_{D}$ equal to two periods. The phase after $2 T_{D}$ differs from the initial phase by a small angle $2 \pi \delta$. This angle is the geometrical view of the detuning. The picture in (a) and (b) does not change if the frequency of the perturbation is close the other parametric resonances $\omega_{S} / \omega_{D}=\frac{1}{2} k$, for $k$ odd. For even $k$ the only difference is that $\phi_{S}$ almost repeats itself after only one interaction.
Eq. (1) changes $\Delta \phi_{S}=\pi+\pi \delta$ for $k=1$ and $\Delta \phi_{S}=\pi \delta(\bmod 2 \pi)$ for $k=0$ for multiples of the driving period $T_{D}$. Therefore the perturbation $P_{n}^{* *}$, has the same phase change for each iteration. A simple approximation to this function around the fix point is

$$
P_{n}^{* *} \approx \xi_{n} h_{1}(\pi n \delta)+h_{0}(\pi n \delta), \quad \xi_{n} \in R .
$$

Here $h_{1}$ and $h_{0}$ are period $2 \pi$ functions. In order to obtain an analytically soluble problem, the functions $h_{1}$ and $h_{0}$ are chosen to be

$$
\begin{aligned}
& h_{1}(\pi n \delta)=(-1)^{n k} A_{1} e^{i \pi \delta}, \\
& h_{0}(\pi n \delta)=(-1)^{n k} A_{0} e^{i \pi n \delta},
\end{aligned}
$$

where $A_{1}$ and $A_{0}$ are real constants. Although the preceding functions are complex, this is done for convenience; the results should be interpreted as "the real part of' the obtained numbers. It is seen that $h_{0}$, because of the factor $n$ in the exponent, has the periodicity already described, whereas $h_{1}$ does not depend on $n$ and is a constant. There are two typical time scales in this problem: one $t_{1}$, of the order $T_{D}$, determines the fast variations between two iterations; the other $t_{0}$ corresponds to the slow variations, changes that occur over a time scale of the or$\operatorname{der} T_{D} / \delta$. Since $t_{1} \ll t_{0}$ for small $\delta$, a constant, $n$ independent of $h_{1}$, can be justified, since the change in $h_{1}$ is essentially adiabatic (i.e., many iterations are necessary in order to observe a sizable change in the $\xi$ independent part of $P_{n}^{* *}$. The constants $A_{0}$ and $A_{1}$ are small compared to 1 and are assumed to be proportional to the amplitude of the perturbation $s(t)$ in Eq. (1). The function $P_{n}^{* *}$ becomes

$P_{n}^{* *} \approx(-1)^{n k} A_{1} e^{i \pi \delta} \xi_{n}+(-1)^{n k} A_{0} e^{i \pi n \delta}, \quad \xi_{n} \in C$.

The variable $\xi$ is now complex. By combining the result of the linearized unperturbed Poincaré map, Eq. (3), with Eq. (7c), one obtains the basic equation

$$
\begin{aligned}
\xi_{n+1}= & {\left[\lambda+(-1)^{n k} A_{1} e^{i \pi \delta}\right] \xi_{n} } \\
& +(-1)^{n k} A_{0} e^{i \pi n \delta}, \quad \xi_{n} \in C .
\end{aligned}
$$

This equation is, for $\delta=0$ and $k=1$, identical to the one derived previously ${ }^{7}$ by the authors. The two constants $A_{0}$ and $A_{1}$ are in general expected to depend on the parameters of the system, e.g., the frequencies of the driving term and the signal, but this dependence is not generally known and is not necessary to understand the main features of the results.

\section{B. The dynamical behavior around the fix point}

From Eq. (8), it is possible to find the influence of perturbations on the period-doubling bifurcation. The main problem is to find solutions to this equation. As seen in Eq. (8), the last term changes in each iteration, and there is no longer a fix point, as was the case in the linearized Poincaré map, Eq. (3). The last term in Eq. (8) can be interpreted as a driving term, so instead of having a single fix point, the attractor is a circle in the complex plane. For every other iteration the point on the attractor slow- 
ly rotates by an angle $2 \pi \delta$. Therefore, it is possible to remove the rotation in the complex plane by applying the following transformation:

$$
\eta_{n}=\xi_{n} e^{-i \pi n \delta} \text {. }
$$

The new coordinate $\eta$ rotates backwards so as to compensate the rotation in $\xi$; it is the equivalent of a "rotating frame." Equation (8) therefore transforms into

$\eta_{n+1}=\left[\lambda e^{-i \pi \delta}+(-1)^{n k} A_{1}\right] \eta_{n}+(-1)^{n k} A_{0} e^{-i \pi \delta}$.

This equation can easily be solved:

$$
\begin{aligned}
& \eta_{\mathrm{even}}^{\mathrm{fix}}=\frac{1+A_{1}-\lambda e^{-\imath \pi \delta}}{\lambda^{2} e^{-2 i \pi \delta}-\left(1+A_{1}^{2}\right)} A_{0} e^{-i \pi \delta}, \\
& \eta_{\mathrm{odd}}^{\mathrm{fix}}=\frac{1+(-1)^{k} A_{1}-\lambda e^{-i \pi \delta}}{\lambda^{2} e^{-2 i \pi \delta}-\left(1+A_{1}^{2}\right)}(-1)^{k} A_{0} e^{-i \pi \delta} .
\end{aligned}
$$

The (even, odd) index refers to $n$. As just seen there is one fix point for $k=0$, and two for $k=1$. In the latter case the iterates alternate between the two fix points. The solution to the original equation, Eq. (8), can be found by applying the inverse of the transformation in Eq. (9), thereby obtaining

$$
\xi_{n}=\eta_{\text {even,odd }}^{\mathrm{fix}} e^{l \pi n \delta}
$$

where the lower index refers to the two possible solutions.

Figure 3(a) shows an example where successive iterates of Eq. (10) are plotted, for $k=1$. In agreement with the solution of Eq. (11) - which corresponds to the rotating frame-the iterates relax along logarithmic spirals towards the two fix points. Figure 3(b) shows the successive iterates of Eq. (8) - in the original frame. Here the iterates relax towards a circle, indicating the $2 \pi \delta$ rotation for every other iteration. It should be noted from Eqs. (11) and (12) that the iterates lie on two circles with slightly different radii, one for even and one for odd iterates. However, as seen from Eq. (11), the difference is of second order in $A_{0}$ and $A_{1}$ and tends to zero for small values of $A_{0}$ and $A_{1}$.

The dynamics around the fix point in the Poincare map is revealed by projecting the dynamics in the complex plane onto the real axis. The attractor in the Poincare map is a line segment just below the bifurcation point. Away from bifurcation the attractor for a system with two frequencies is an ellipse in the Poincare map. When the system is close to a period-doubling bifurcation, however, the dynamics is confined to the center manifold, which in the Poincaré map reduces to a one-dimensional curve (see Fig. 1). The line segment is therefore an elongated ellipse. After bifurcation is established, the line segment splits into two line segments: that is what bifurcation in fact means. A word of caution is necessary: it is not easy to visualize the dynamics both in phase space and in the complex plane at the same time (which would require four-dimensional images). The circle in the complex plane is unrelated to the mentioned ellipse in the Poincaré map.

\section{The stability of the period-doubling bifurcation}

The stability of the fix points must now be examined. This is very important, since it reveals one of the effects of a periodic perturbation, namely, the shift in the bifurcation point. The stability can be investigated by looking at the asymptotic behavior around a given fix point. The deviation from the fix point is defined as

$$
\Delta \eta_{n}=\eta_{n}-\eta^{\mathrm{fix}},
$$

which leads to the following equation for the asymptotic
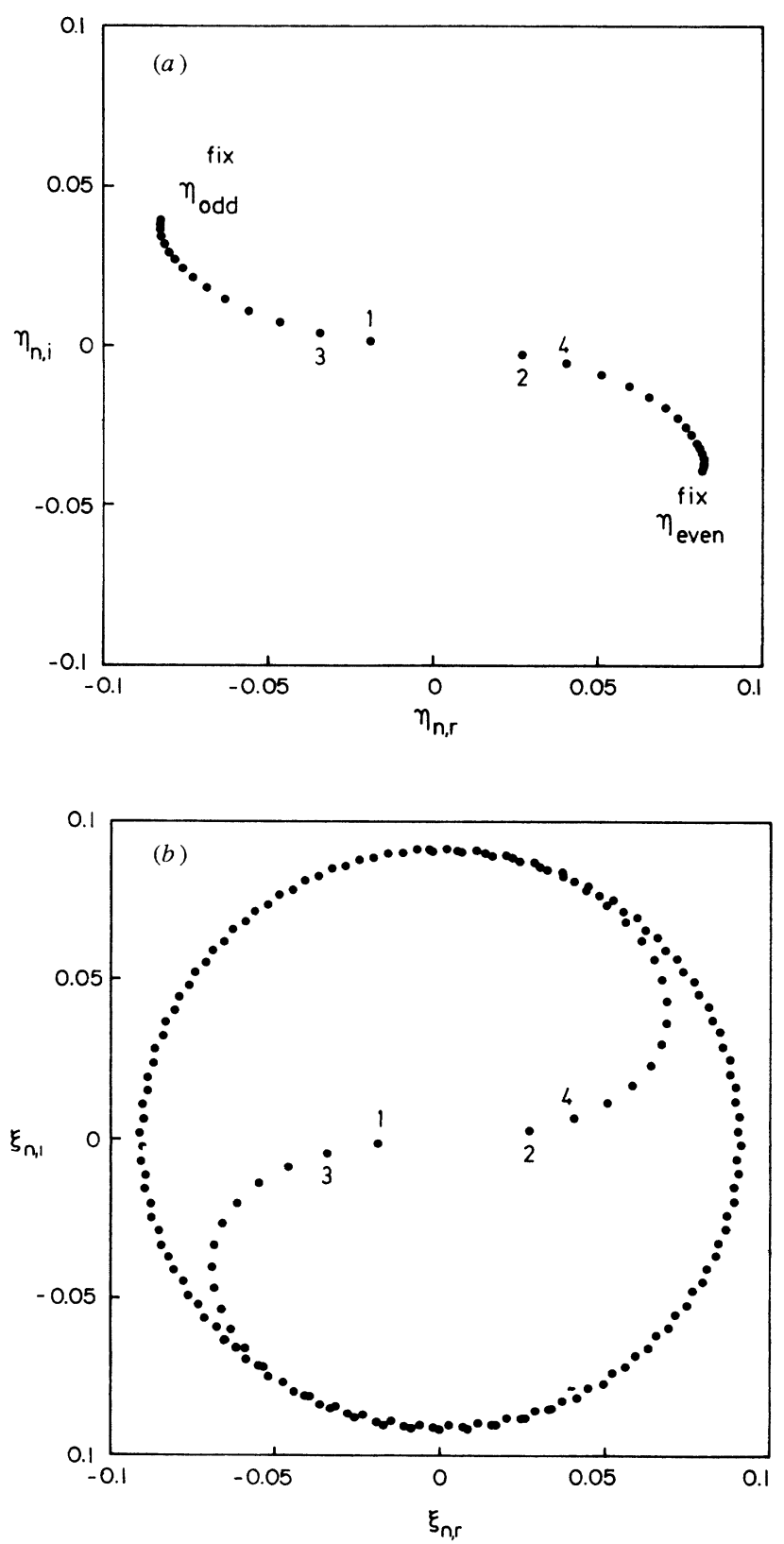

FIG. 3. The successive iterations towards the fix points in the complex plane [Eqs. (8) and (10)] for $\lambda=-0.9, k=1$, $A_{0}=A_{1}=0.01$, and $\delta=\frac{1}{64}$. (a) shows how $\xi_{n}$ relaxes towards the two fix points according to Eq. (10); (b) shows the iteration of the original Eq. (8). 
behavior

$$
\Delta \eta_{n+1}=\left[\lambda e^{-i \pi \delta}+(-1)^{n k} A_{1}\right] \Delta \eta_{n} .
$$

The two cases $k=0$ and $k=1$ are treated separately.

(1) For $k=0$. The fix point of Eq. (11) is stable if

$$
\left|\lambda e^{-i \pi \delta}+A_{1}\right|<1 .
$$

At the limit of stability the left-hand side is equal to 1 . Therefore,

$$
-\lambda-A_{1} \cos \pi \delta \approx 1 .
$$

For $\lambda=-1-\mu$, where $\mu$ is the control parameter near the bifurcation, an equation for the shift of the bifurcation point is obtained:

$$
\Delta \mu_{B} \propto A_{1} \cos \pi \delta,
$$

where $A_{1}$ is assumed to be proportional to the amplitude of the periodic perturbation, and the change in stability depends therefore on the sign of the amplitude and thereby on the instantaneous phase difference between the perturbation and the drive. For small $\delta$ the variation of $h_{1}$ is very slow; therefore the preceding result, Eqs. (15), is the adiabatic limit. For small $\delta$ the sign of $A_{1}$ does change for every half period of $h_{1}$ [Eq. (7b)]. The system, therefore, may slowly drift in and out of bifurcation with a frequency of $2 \pi \delta$. There is a change of the stability condition-although not a change with a well-defined sign - when the frequency of the perturbation is close to the frequency of the drive, $\omega_{S} / \omega_{D} \approx 1$. The shift in the value of the control parameter $\mu$ at bifurcation can be decreased by increasing the value of the detuning $\delta$, according to Eq. (15b).

(2) For $k=1$. In this case the sign of $A_{1}$ changes for each iteration. Therefore, the stability has to be investigated for every other iteration, which leads to the equation

$$
\Delta \eta_{n+2}=\left(\lambda^{2} e^{-i 2 \pi \delta}-A_{1}^{2}\right) \Delta \eta_{n}
$$

The criterion for stability becomes

$$
\left|\lambda^{2} e^{-i 2 \pi \delta}-A_{1}^{2}\right|<1 \text {. }
$$

Once again at the limit of stability

$$
\lambda^{2}-A_{1}^{2} \cos 2 \pi \delta \approx 1,
$$

which leads to the following equation for the shift of the control parameter at the bifurcation point:

$$
\Delta \mu_{B} \propto \frac{1}{2} A_{1}^{2} \cos 2 \pi \delta \text {. }
$$

For small $\delta$

$$
\Delta \mu_{B} \propto \frac{1}{2} A_{1}^{2} \text {. }
$$

This equation gives a second important result: When the periodic perturbation is close in frequency to half the driving frequency, $\omega_{S} / \omega_{D} \approx \frac{1}{2}$, the periodic perturbation always stabilizes the system against a period-doubling bifurcation. The shift in the control parameter at the bifurcation point is always positive, and proportional to the square of the amplitude of the perturbation. The detun- ing decreases the effect according to Eqs. (18). Once again the results in Eqs. (18) are valid for small $\delta$, i.e., in the adiabatic limit. A slow modulation of $A_{1}$, results in the convergence also exhibiting a slow modulation. ${ }^{14}$

\section{Amplification of perturbations}

Another important property of a period-doubling bifurcation is its ability to amplify perturbations. In the case of a periodic perturbation this can be seen from Eq. (11). As the control parameter approaches the bifurcation point, the fix points (Eqs. (11)] move away from the original unperturbed fix point, which corresponds to a dynamic amplification - a resonance-in the dynamical system. This resonance can be found in Eq. (11) as the minimum of the magnitude of the denominator-which also corresponds to the real part of the denominator becoming zero. It yields

$$
\lambda^{2}=\left(1+A_{1}^{2}\right) \cos 2 \pi \delta .
$$

which, for small $\delta$, becomes

$$
\Delta \mu_{R} \propto \frac{1}{2} A_{1}^{2}-(\pi \delta)^{2} .
$$

The subindex on $\mu_{R}$ indicates the value of the control parameter at resonance. This equation shows that the control parameters $\mu$ at resonance differ from the unperturbed bifurcation value by an amount that is a function of both $A_{1}$ and $\delta$. Comparison of the control parameters at bifurcation and resonance, $\mu_{B}$ and $\mu_{R}$, give the inequality

$$
\Delta \mu_{B} \geq \Delta \mu_{R} .
$$

This inequality shows that the resonance maximum (or maximum gain point) always appears before the bifurcation, as the control parameter is increased. It also indicates that, in general, it is almost impossible to find the actual bifurcation point in a frequency spectrum, since the resonance effects dominate in the spectrum. ${ }^{6}$ For zero detuning, $\delta=0$, the bifurcation and maximum resonance (maximum gain) points coincide.

\section{E. Inclusion of noise}

Noise in the system [the term $v(t)$ in Eq. (1)] can be modeled by adding a noise term $v_{n}$ in Eq. (8), which produces fluctuations in $\xi_{n}$. The noise is characterized by

$$
\left\langle v_{n} v_{m}\right\rangle=\sigma^{\prime} \delta_{n m},\left\langle v_{n}\right\rangle=0 \text {. }
$$

This is equivalent to the addition of a noise term $v_{n}^{\prime}$ in Eq. (10) for $\eta_{n}$; the spectral conditions for $v_{n}^{\prime}$ are identical to those for $v_{n}$, Eq. (22). This noise gives rise to fluctuations in $\Delta \eta_{n}$, which, in turn, yield random intersections of the trajectory in the Poincare map. The fluctuations are assumed to be restricted to the center manifold, i.e., one-dimensional noise in the Poincaré map.

Once again the two cases $k=0$ and $k=1$ must be considered separately.

(1) For $k=0$. Here the behavior of the bifurcation [Eqs. (15)] and the resonance [Eqs. (20)] are quite different. For $m>n$ the autocorrelation function of $\Delta \eta_{n}$ 
become

$$
\left\langle\Delta \eta_{m}^{*} \Delta \eta_{n}\right\rangle=\frac{\sigma^{\prime}\left(\lambda e^{i \pi \delta}+A_{1}\right)^{m-n}}{1-\left(\lambda^{2}+A_{1}^{2}+2 \lambda A_{1} \cos \pi \delta\right)},
$$

which diverges at the bifurcation point given by Eqs. (15).

(2) For $k=1$. In this more interesting case the behavior of Eqs. (18) and Eqs. (20) are quite similar. For $m>n$ and $m-n$ even, the autocorrelation function of $\Delta \eta_{n}$ becomes

$$
\begin{aligned}
\left\langle\Delta \eta_{n}^{*} \Delta \eta_{m}\right\rangle= & \frac{\sigma^{\prime}\left[1+\lambda^{2}+A_{1}^{2}-(-1)^{n} 2 A_{1} \lambda \cos \pi \delta\right]}{1-\left(\lambda^{4}+A_{1}^{4}-2 \lambda^{2} A_{1}^{2} \cos 2 \pi \delta\right)} \\
& \times\left(\lambda^{2} e^{-2 \pi i \delta}-A_{1}^{2}\right)^{(n-m) / 2}
\end{aligned}
$$

which diverges at the bifurcation point given by Eqs. (18).

Thus the noise is also amplified as the bifurcation point is approached. ${ }^{15}$ It is important to note that the noise does not influence the stability of the system, only the periodic perturbation has this effect. This conclusion is of course based on the particular way noise is modeled in here, namely, as a additive uncorrelated noise with zero mean. Other work on this problem has found that noise may influence the stability, ${ }^{16,17}$ but in that work the bifurcation point is defined from changes in the distribution function of the Poincaré map.

\section{NUMERICAL AND ANALOG SIMULATIONS}

In order to illustrate some of the features of the theory just presented, the differential equation describing a microwave-driven Josephson junction was solved both numerically and by analog simulation. The governing differential equation is ${ }^{18}$

$$
\phi_{t t}+\alpha \phi_{t}+\sin \phi=a_{D} \sin \omega_{D} t+a_{S} \sin \omega_{S} t+\eta .
$$

Here $\phi$ is the quantum-mechanical phase difference across the junction and $\alpha$ is the damping parameter $\left(\alpha=1 / \sqrt{\beta_{C}}\right.$, where $\beta_{C}$ is the McCumber parameter for the junction). The quantities $a_{D}=A_{D} / I_{C}$ and $a_{S}=A_{S} / I_{C}$ are the normalized amplitudes of the driving and resonant perturbing signals (normalized to the critical current of the junction, $\left.I_{C}\right) ; \omega_{D}$ and $\omega_{S}$ are the corresponding frequencies [normalized to the junction maximum plasma frequency $\omega_{p}=\left(2 e I_{C} / h C\right)^{1 / 2}$, where $C$ is the junction capacitance]; $\eta=I_{D C} / I_{C}$ is the normalized dc bias. It should be noted that this equation also describes a driven, damped pendulum subject to two periodic perturbing forces.

In order to integrate Eq. (25) numerically, a fourthorder Runge-Kutta method with 32 points per period of the driving signal was used. The motion of $\phi$ was followed over 256 periods of the drive and the first 128 periods were discarded in order to remove transients. Throughout the calculations the following fixed parameter values were used: $\alpha=0.2, \omega_{D}=1.6$, and $\eta=0.73$; the parameters $a_{D}$ and $a_{S}$ were varied. The amplitude of the driving force, $a_{D}$, was chosen as the control parameter $\mu$ of the system: its value was such that the system was near a period-doubling bifurcation.

The results of the digital calculations for $\omega_{S} / \omega_{D} \approx \frac{1}{2}$ and three different values of $\delta$ are shown in Fig. 4. The

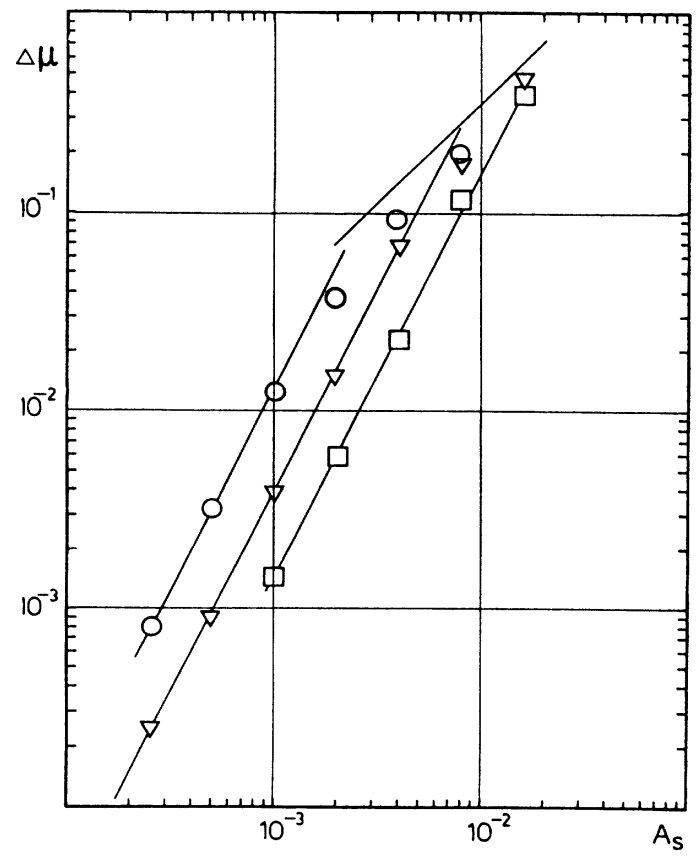

FIG. 4. Results of numerical calculations for the change in the control parameter $\Delta \mu$, necessary to reach the bifurcation point, as a function of the perturbation amplitude $A_{S}$ for three values of the detuning: circles correspond to $2 \delta / \omega_{D}$ equal to $-\frac{1}{256}$; triangles correspond to $2 \delta / \omega_{D}$ equal to $-\frac{1}{128}$; and squares correspond to $2 \delta / \omega_{D}$ equal to $-\frac{1}{64}$. The fitted straight lines in the logarithmic graph have slopes of 2 and 1.

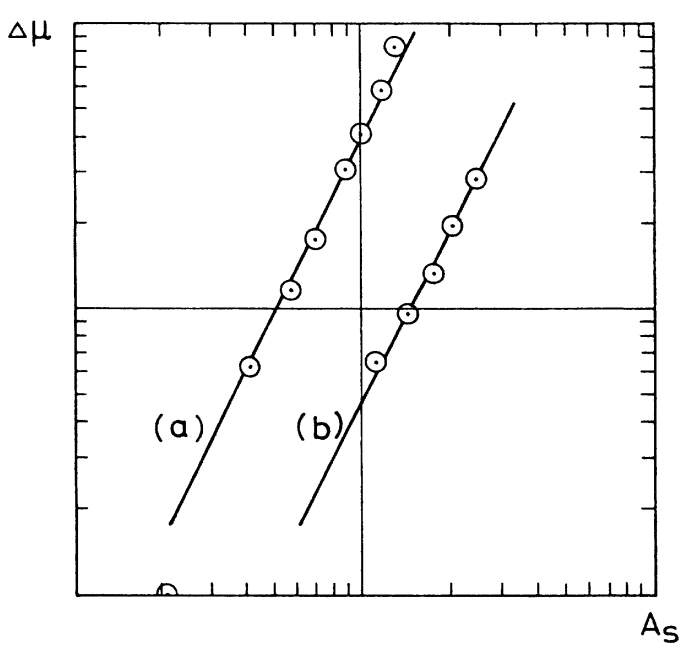

FIG. 5. The quadratic scaling law of Eqs. (18) as investigated by analog simulation. The shift in the bifurcation point is defined as $\Delta \mu=A_{D}-\left(A_{D}\right)_{0}$, where $\left(A_{D}\right)_{0}$ is the pump amplitude at the bifurcation point of the unperturbed system. The figure shows a logarithmic plot of the signal amplitude $A_{S}$ vs $\Delta \mu$. Curve $(a)$ is for $k=1$, i.e., for $\omega_{S} \approx \frac{1}{2} \omega_{D},\left(f_{D}=8021 \mathrm{~Hz}\right.$ and $\left.f_{S}=3955 \mathrm{~Hz}\right)$. Curve $(b)$ is for $k=3$, i.e., for $\omega_{S} \approx \frac{3}{2} \omega_{D}$, $\left(f_{D}=8058 \mathrm{~Hz}\right.$ and $\left.f_{S}=12035 \mathrm{~Hz}\right)$. The solid straight lines have slope 2 , and the circles are the data from the simulation. Both the $\Delta \mu$ and the $A_{S}$ axis are in arbitrary units. 


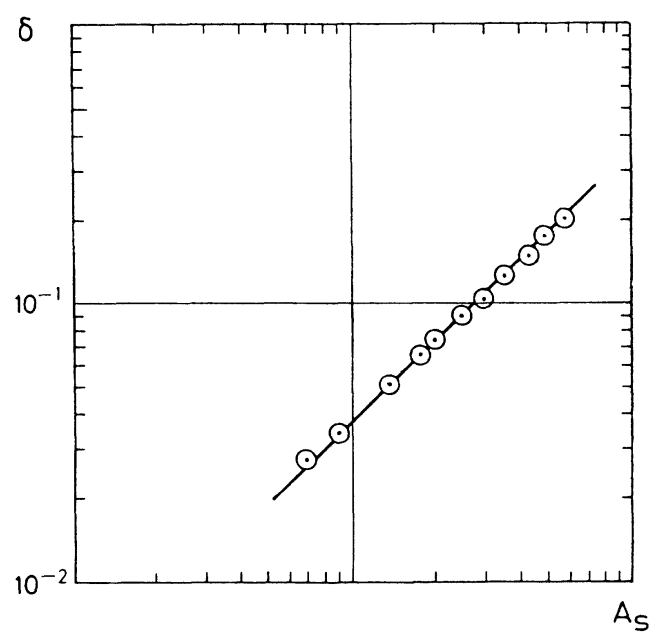

FIG. 6. The values of the detuning $\delta$ and of the input signal amplitude $A_{S}$ for constant amplification of the perturbating signal $A_{S}$. The solid line has slope 1, and the circles are the data from the simulation. The curve is made for $k=1$, i.e., for $\omega_{S} \approx \frac{1}{2} \omega_{D},\left(f_{D}=7987 \mathrm{~Hz}\right)$.

shift of the bifurcation point, as measured by the change in the drive amplitude $a_{D}$, is plotted against the perturbation amplitude $a_{S}$. From Fig. 4 it is seen that for the calculated values the quadratic scaling law predicted by Eq. (20), $\Delta a_{D} \propto a_{S}^{2}$, holds as long as the perturbation amplitude is small. Above a certain level of perturbation, which depends on the relative detuning $\delta$, the relationship between $a_{D}$ and $a_{S}$ becomes almost linear. This deviation from the theory is believed to be caused by nonlinear effects.

Equation (25) was also simulated by using a Magerlein-type analog circuit (described in Ref. 19). The amplitude of the drive $A_{D}$ is taken as the bifurcation parameter $\mu$. In Fig. 5 the quadratic scaling law of Eqs. (18) is shown, both for $k=1$ and for $k=3$, i.e., $\omega_{S} \approx \frac{1}{2} \omega_{D}$ and $\omega_{S} \approx \frac{3}{2} \omega_{D}$. The amplitude of the signal was one order of magnitude larger in the last case. It is not known whether the quadratic scaling law for $\omega_{S} \approx \frac{3}{2} \omega_{D}$ is caused by mixing of frequencies down to $\omega=\omega_{S}-\omega_{D} \approx \frac{1}{2} \omega_{D}$; these frequencies cannot be separated in the nonlinear system. The calculation is in good agreement with the theoretical scaling law of Eqs. (18). By a variation of the input amplitude of the signal $A_{S}$ and of the detuning $\delta$ in such a way that the amplification of the signal is kept constant, it is possible to check Eq. (20b). Figure 6 shows those results. They confirm the prediction of Eqs. (20), i.e., $A_{S} \propto \delta$.

\section{DISCUSSION AND CONCLUSION}

In this paper the influence of perturbations on a dynamical system that is close to undergoing perioddoubling bifurcation has been investigated. The method employed is based on the existence of a center manifold close to the bifurcation. This center manifold makes the dynamics in the Poincare map essentially one dimensional. The dynamics around the unperturbed fix point in the Poincare map was examined. The solution of the resulting equation reveals several interesting features of the perturbed period-doubling bifurcation.

It was found that even the smallest near-resonant periodic perturbations (signals) affect the stability of the system. This stability is not a static property: the convergence changes periodically in time, with a long period of the order $1 / \delta$, where $\delta$ is the frequency detuning. The system may slowly drift in and out of the bifurcation.

It is instructive to compare the criteria of stability with Floquet's theory: ${ }^{20}$ there stability considerations are based on the total period of the system, i.e., the combined period of the drive and the signal $(1 / \delta$, where $\delta$ is now assumed to be rational). Therefore the stability is based on the long period. Even though this period is stable, the results found here show that it is possible for the system to drift in and out of the bifurcation within one such (long) period. Floquet's theory is therefore not a convenient way to examine stability in these conditions.

The amplification of periodic perturbations (signals) is not a steady-state process, since it depends on the stability of the system. Usually the amplification of a signal is determined from a power spectrum, and thus involves averaging over a certain time interval. Therefore periodic changes that take place over time intervals that are small compared to that time are not seen. It is clear, however, that the spectrum must be influenced by the instabilities inherent in the system when it is close to the bifurcation point. For example, the superconducting Josephson junction parametric amplifiers have been plagued by very high noise levels in the amplifying mode; the dynamical amplification discussed in this paper may have an influence on the noise level.

The theory presented here also provides a scaling law for the shift in the bifurcation point. It was found that the change in the control parameter at bifurcation $\Delta \mu$ is proportional to $\left(A_{S}\right)^{2}$, the square of the signal amplitude, for $\omega_{S} \simeq \frac{1}{2} \omega_{D}$. It was also discovered that the amplification is a function of the ratio $A_{S} / \delta$; i.e., for constant amplification the signal amplitude and the detuning parameter are proportional to each other. These two scalings law were investigated by numerical and analog simulations and found to be in good agreement with the theory presented here (see Figs. 4-6). Also experiments on Josephson junctions have confirmed these scaling laws. ${ }^{13}$

An interesting feature of the theory is that it is linear in the perturbations. Therefore the effects found in this paper are not in the lowest-order nonlinear effects, in contrast to what has been believed earlier. ${ }^{4}$ Nonlinear effects are important for large perturbations, but in that case no definite scaling laws (such as those for the shift of the bifurcation point) are expected to exist. The theory presented here of course breaks down for large perturbations.

\section{ACKNOWLEDGMENTS}

The authors would like to acknowledge the hospitality of the Department of Physics, University of California, 
Berkeley. They would like to thank L. M. Falicov for stimulating discussions. One of us (H.S.) would also like to acknowledge the financial support from Carlsberg fondet (Denmark); Fabriksejer, Ingenior Valdemar Selmer Trane og Hustru Elisa Tranes Fond (Denmark); the Danish-American Foundation; the Knud Hojgaard Fond (Denmark); Nato Fellowship Science Programme; Danish
Natural Science Research Council (Denmark); Otto Mønsteds Fond (Denmark); and Thomas B. Thriges Fund (Denmark). This research was supported, at the Lawrence Berkeley Laboratory, by the Director, Office of Energy Research, Office of Basic Energy Sciences, Materials Sciences Division, U.S. Department of Energy, under Contract No. DE-AC03-76SF00098.
${ }^{1}$ J. Heldstab, H. Thomas, T. Geisel, and G. Radons, Z. Phys. B 50, 141 (1983).

${ }^{2}$ K. Wiesenfeld and B. McNamara, Phys. Rev. Lett. 55, 13 (1985).

${ }^{3}$ K. Wiesenfeld and B. McNamara, Phys. Rev. A 33, 629 (1986).

${ }^{4}$ P. Bryant and K. Wiesenfeld, Phys. Rev. A 33, 2525 (1986).

${ }^{5}$ K. Wiesenfeld, J. Stat. Phys. 28, 1071 (1985).

${ }^{6}$ H. Svensmark, J. Bindslev Hansen, and N. F. Pedersen, Phys. Rev. A 35, 1457 (1987).

${ }^{7}$ H. Svensmark and M. R. Samuelsen, Phys. Rev. A 36, 2413 (1987).

${ }^{8}$ M. J. Feldman, P. T. Parrish, and R. Y. Chiao, J. Appl. Phys. 46, 4031 (1975).

${ }^{9}$ Y. Taur and.P. L. Richards, J. Appl. Phys. 48, 1321 (1977).

${ }^{10} \mathrm{~J}$. Mygind, N. F. Pedersen, O. H. Sorensen, B. Dueholm, and M. T. Levinsen, Appl. Phys. Lett. 35, 91 (1979).

${ }^{11}$ M. T. Levinsen, N. F. Pedersen, O. H. Sorensen, B. Dueholm, and J. Mygind, IEEE Trans. Electron Devices ED-27, 1928 (1980).

12J. Guckenheimer and P. Holmes, Nonlinear Oscillations,
Dynamical Systems and Bifurcations of Vector Fields (Springer, New York, 1984).

${ }^{13}$ G. F. Eriksen and J.B. Hansen, following paper, Phys. Rev. B 41, 4188 (1990).

${ }^{14} \mathrm{By}$ numerically solving the Mathieu equation, with a small parametric perturbation corresponding to $\omega_{S} \approx \frac{1}{2} \omega_{D}$, two features where noted: (1) the signal had a stabilizing effect, and (2) the stability (convergence) was different in the two orthogonal phases.

${ }^{15}$ The influence of noise alone in this model was examined in Ref. 7.

${ }^{16}$ W. Horsthemke and M. Malek-Mansour, Z. Phys. B 24, 307 (1976).

${ }^{17}$ K. H. Hoffmann, Z. Phys. B 49, 245 (1982).

${ }^{18}$ For a good description of the Josephson-junction equation see, for example, A. Barone and G. Paterno, Physics and Applications of the Josephson Effect (Wiley, New York, 1982).

${ }^{19}$ J. H. Magerlein, Rev. Sci. Instrum. 49, 886 (1978).

${ }^{20}$ D. W. Jordan and P. Smith, Nonlinear Ordinary Differential Equations (Oxford University Press, Oxford, 1977). 